\title{
Zur Verleihung des Abel-Preises 2007 an S. R. Srinisava Varadhan
}

Erwin Bolthausen und Alain-Sol Sznitman

Varadhan ist der erste Wahrscheinlichkeitstheoretiker, der den Abel-Preis erhielt. Er hat die Wahrscheinlichkeit in ihrer heutigen Form tief und nachhaltig geprägt. Er hat neue Konzepte eingeführt, die die Lösung schwieriger Probleme ermöglicht haben und die anderseits der Theorie ganz neue Richtungen und Entwicklungen gegeben haben. Sein Werk ist aufs engste mit den Theorien der Diffusionsprozesse, der großen Abweichungen, der zufälligen Medien und der stochastischen Vielteilchensysteme verknüpft.

Ende der Sechzigerjahre und zu Beginn der Siebziger hat Varadhan zusammen mit Daniel Stroock einen neuen Zugang zur Theorie der Diffusionsprozesse entwickelt, indem diese stochastischen Prozesse über das so genannte Martingalproblem charakterisiert werden. Damit wurde eine weitreichende Verallgemeinerung von Paul Lévys Martingalcharakterisierung der Brownschen Bewegung erreicht. Der neue Zugang beschreibt Diffusionsprozesse als schwache Lösungen einer stochastischen Differentialgleichung, die dadurch eindeutig beschrieben werden, dass gewisse Transformationen der Prozesse Martingale sind. Im Vergleich zum älteren Itôschen Zugang ist diese Methode hervorragend geeignet, Grenzwertsätze in Form von Invarianzprinzipien zu beweisen, mit denen etwa die Konvergenz von Markoffschen Ketten gegen Diffusionsprozesse behandelt werden kann, oder Homogenisierungsprobleme bei Prozessen mit stark oszillierenden Koeffizienten. Die Formulierung als Martingalproblem gehört heute zu den Standardmethoden in der Theorie Markoffscher Prozesse, die sich insbesondere auch für die Konstruktion unendlichdimensionaler Prozesse als sehr fruchtbar erwiesen hat.
Fast ebenso wichtig und einflussreich sind die Arbeiten von Stroock und Varadhan zu Supportproblemen für Diffusionsprozesse. In ihnen wird der Träger des Maßes auf dem Pfadraum als Abschluss einer Familie von Lösungen von deterministischen Differentialgleichungen beschrieben. Damit verbunden ist der Nachweis von Stetigkeitseigenschaften, mit denen die Reaktion eines stochastischen Systems auf zugefügtes Rauschen untersucht werden kann. Dies war ein bedeutender Schritt in Richtung des später entwickelten Malliavin-Kalküls, für den diese Fragen von zentraler Bedeutung sind.

In einem engem Zusammenhang mit diesen neuen Entwicklungen zu Diffusionsprozessen standen die ersten Arbeiten von Varadhan zur Theorie großer Abweichungen. Er untersuchte das Verhalten des Wärmeleitungskerns elliptischer Operatoren für kleine Zeit und zeigte, dass ihre Asymptotik außerhalb der Diagonalen durch das Verhalten des Diffusionsprozesses in einem „untypischen“ Bereich des Pfadraums beschrieben werden kann. Dabei stieß er auf die wichtige Rolle, die gewisse quadratische Aktionsfunktionale spielen, welche durch eine Riemannsche Metrik determiniert sind. Diese Metrik wird durch die Inverse der Matrix der Diffusionsmatrix bestimmt. Die Arbeiten bildeten den Ausgangspunkt zu sehr detaillierten Untersuchungen des Wärmeleitungskerns durch andere Autoren wie Bismut, Stroock, Ben Arous, Kusuoka oder Léandre. Noch bedeutsamer war freilich, dass damit der Grundstein zu einer sehr weit reichenden und tiefen allgemeinen Theorie von Wahrscheinlichkeiten großer Abweichungen gelegt wurde. Varadhan hat diese etwa zehn Jahre später zusammen mit Monroe Donsker zu einer kohärenten sehr allgemeinen Methode entwickelt, indem 


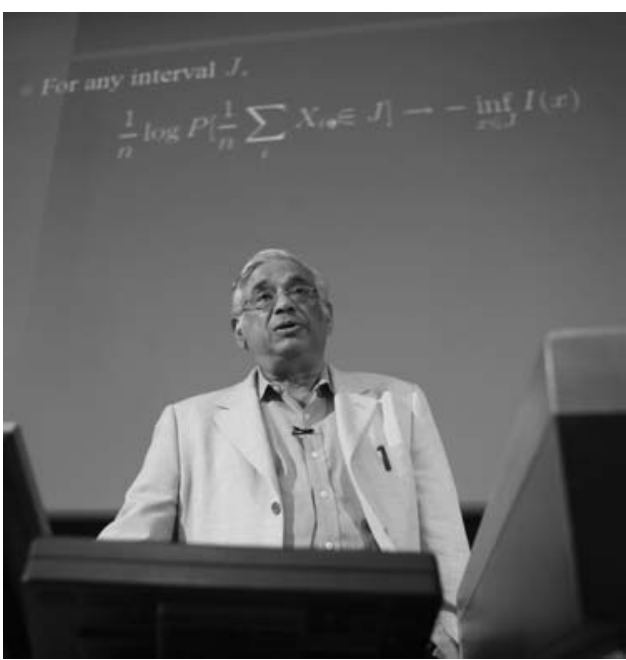

S. R. Srinivasa Varadhan bei der Abel Lecture an der Universität Oslo (Photo: Terje Bendiksby/Scanpix/The Abel Prize/The Norwegian Academy of Science and Letters)

sie eine Untersuchung der großen Abweichungen des Langzeitverhaltens von Markoffschen Prozessen in die Wege leiteten. Die grundlegende Idee besteht darin, die „Kosten“ präzise zu beschreiben, die ein vom ergodischen abweichendes Verhalten verursacht. Wie sich herausstellte, war es sowohl konzeptuell wie für konkrete Anwendungen zweckmäßig, sehr hochdimensionale Konstrukte zu untersuchen, was schlussendlich zu einer Theorie großer Abweichungen von empirischen Prozessen des vollständigen Pfades führte. Die niedrigdimensionalen Abweichungen konnten anschließend über so genannte Kontraktionsprinzipien gewonnen werden. Besonders bemerkenswert war, dass mit dieser sehr abstrakten Theorie im Wechselspiel zwischen hoch- und niedrigdimensionalen Gesichtspunkten sehr konkrete und schwierige Probleme gelöst wurden, wie die Pekar-Vermutung aus der Quantenstatistik oder eine Vermutung von Kac und Luttinger über die Umgebung der Spur eines Brownschen Pfades, die eng mit zufälligen Schrödingeroperatoren zusammenhängt.

Ein anderes Forschungsgebiet Varadhans war die Theorie von Irrfahrten und Diffusionen in zufälligen Medien. In klassischen Homogenisierungsproblemen wird das asymptotische Diffusionsverhalten in räumlich periodischen $\mathrm{Me}$ dien betrachtet. Im stochastischen Fall hingegen untersucht man stattdessen zufällige ,verrauschte" Diffusionsparameter oder Irrfahrten mit zufälligen Übergangswahrscheinlichkeiten. Die Behandlung solcher Medien erforderte ein völlig neues Konzept, in welchem die für das makroskopische Verhalten relevante kurzreichweitig fluktuierende Umgebung als eine Verschiebung des zufälligen Mediums durch die makroskopische Position aufgefasst werden musste. Dieses Konzept wurde unabhängig durch Sergei Kozlov in Russland und in ei- ner Arbeit von Papanicolaou und Varadhan um 1980 entwickelt. Es ist heute unter dem Schlagwort random environment viewed from the particle bekannt und ist eines der wichtigsten in diesem Gebiet. Um den Einfluss der mikroskopischen Umgebung diskutieren zu können war es wichtig, für diesen hochdimensionalen Prozess ein invariantes $\mathrm{Ma}$ mit gewissen ,guten" Eigenschaften zu finden. Ein entscheidender Fortschritt wurde durch eine Arbeit von Claude Kipnis und Varadhan erreicht, in der dieses Programm erfolgreich durchgeführt werden konnte, sofern gewisse Reversibilitätsannahmen erfüllt sind. Damit war der Grundstein zu vielen Entwicklungen der letzten Jahre in einer großen Reihe von physikalisch relevanten Anwendungen gelegt.

Ebenso einflussreich für die wahrscheinlichkeitstheoretische Forschung waren die Beiträge Varadhans ab Ende der Achtzigerjahre zu Problemen in der Statistischen Mechanik über den Zusammenhang zwischen dem mikroskopischen und makroskopischen Verhalten großer Systeme von stochastischen Vielteilchensysteme. Eine notorisch schwierige und fundamentale Frage betrifft die makroskopische Beschreibung eines Systems durch Differentialgleichungen, z. B. in Form der Eulerschen Gleichungen in der Hydrodynamik, ausgehend von der Beschreibung auf mikroskopischer Ebene durch die Hamiltonschen Gleichungen. Ähnliche Fragen treten in vielen anderen Modellen auf, wie zum Beispiel bei Ausschlussprozessen und in der Ginsburg-Landau-Theorie. Der Kern des Problems besteht darin, die zeitliche Entwicklung von lokalen Erhaltungsgrößen präzise zu beschreiben. Diese sich langsam verändernden Größen werden nach einer geeigneten Skalierung durch die makroskopischen Gleichungen beschrieben. Die Schwierigkeit besteht darin, die lokalen Größen richtig zu mitteln. Entscheidend ist, dass sich das System lokal genähert in einem Gleichgewichtszustand befindet, welches durch ein Gibbs-Maß mit nur großräumig sich ändernden Parametern beschrieben wird. Die Abkopplung der langsamen globalen Bewegung von der schnellen lokalen erfordert eine außerordentlich präzise Kontrolle des Gesamtsystems. Besonders beeindruckend waren die Resultate zu so genannten Nichtgradientensystemen, denen eine spezielle Kürzungseigenschaft der Gradientensysteme abgeht. Varadhans Beiträge zu diesem Gebiet haben ein eigenständiges Forschungsgebiet neu begründet, das weiterhin zu den aktuellsten und aktivsten der Wahrscheinlichkeitstheorie gehört.

Die Resultate dieser stochastischen Theorie sind von großem Interesse auch in der Theorie deterministischer Differentialgleichungen. Es hat sich nämlich gezeigt, dass die genaue stochastische Modellierung mit schwierigen Fra- 
gen über die Existenz und Eindeutigkeit der makroskopischen Gleichung zusammenhängt, wie etwa der richtigen Auswahl der Lösung über Entropiebedingungen.

Zum Schluss noch einige biographische Details: S. R. Srinisava Varadhan wurde 1940 in Madras (Indien) geboren. Er promovierte 1963 beim Statistiker C. R. Rao am Indian Statistical Institute in Calcutta. Danach erhielt er eine Postdoktorandenstelle am Courant-Institut in New York, an dem er seither mit Unterbrechungen gearbeitet hat. 1966 wurde er Assistenzprofessor, 1968 Associate Professor und 1972 Full Professor, stets am Courant-Institut. Schon bevor er den Abel-Preis erhielt, wurde er mit einer Vielzahl von Preisen und Auszeichnungen geehrt, so 1996 mit dem AMS Steele Prize und 2003 mit dem Ehrendoktorat der renommierten Pariser Universität Pierre et Marie Curie.

Varadhan ist verheiratet und hat einen Sohn. Sein ältester Sohn kam beim Terroranschlag am II. September 200I auf die Zwillingstürme in Manhattan tragisch ums Leben.

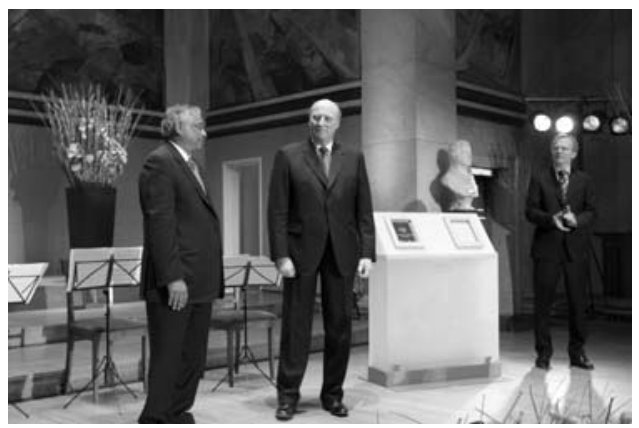

König Harald verleiht S.R. Srinivasa Varadhan den Abel-Preis. Rechts: Kristian Seip, Chairman des AbelKomitees. (Photo: Terje Bendiksby/Scanpix/The Abel Prize/The Norwegian Academy of Science and Letters)

\section{Adresse der Autoren}

Prof. Dr. Erwin Bolthausen

Institut für Mathematik

Universität Zürich

Winterthurerstraße 190

8057 Zürich

Schweiz

eb@math.unizh.ch

Prof. Dr. Alain-Sol Sznitman

Departement Mathematik

HG G 36.2

ETH Zürich

Rämistraße I0I

8092 Zürich

Schweiz

alain-sol.sznitman@math.ethz.ch

Viele weitere interessante Informationen zu Leben und Werk von S. R. Srinivasa Varadhan können Sie in Heft 65 des Newsletter of the European Mathematical Society in einem Interview finden, das Martin Raussen und Christian Skau mit ihm durchgeführt haben.

Den Newsletter bekommen alle persönlichen Mitglieder der EMS (Mitgliedschaft über die DMVGeschäftsstelle, Jahresbeitrag 20 Euro), im Internet ist er unter http://www.ems-ph.org/newsletter/ news.php zu finden. 